Suter, Claudia; Högger, Dominique

\title{
Ernährungsbildung - was sollen, dürfen und müssen Lehrerinnen und Lehrer?
}

Haushalt in Bildung \& Forschung 3 (2014) 4, S. 16-27

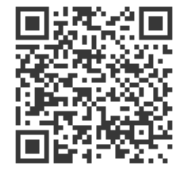

Quellenangabe/ Reference:

Suter, Claudia; Högger, Dominique: Ernährungsbildung - was sollen, dürfen und müssen Lehrerinnen und Lehrer? - In: Haushalt in Bildung \& Forschung 3 (2014) 4, S. 16-27 - URN: urn:nbn:de:0111-pedocs-204316 - DOI: 10.25656/01:20431

https://nbn-resolving.org/urn:nbn:de:0111-pedocs-204316

https://doi.org/10.25656/01:20431

in Kooperation mit / in cooperation with:

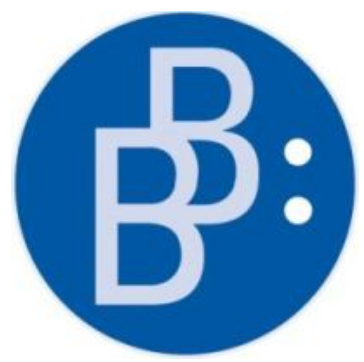

https://www.budrich.de

\section{Nutzungsbedingungen}

Gewährt wird ein nicht exklusives, nicht übertragbares, persönliches und beschränktes Recht auf Nutzung dieses Dokuments. Dieses Dokument ist ausschließlich für den persönlichen, nicht-kommerziellen Gebrauch bestimmt. Die Nutzung stellt keine Übertragung des Eigentumsrechts an diesem Dokument dar und gilt vorbehaltlich der folgenden Einschränkungen: Auf sämtlichen Kopien dieses Dokuments müssen alle Urheberrechtshinweise und sonstigen Hinweise auf gesetzlichen Schutz beibehalten werden. Sie dürfen dieses Dokument nicht in irgendeiner Weise abändern, noch dürfen Sie dieses Dokument für öffentliche oder kommerzielle Zwecke vervielfältigen, öffentlich ausstellen, aufführen, vertreiben oder anderweitig nutzen.

Mit der Verwendung dieses Dokuments erkennen Sie die Nutzungsbedingungen an.

\section{Terms of use}

We grant a non-exclusive, non-transferable, individual and limited right to using this document.

This document is solely intended for your personal, non-commercial use. Use of this document does not include any transfer of property rights and it is conditional to the following limitations: All of the copies of this documents mus retain all copyright information and other information regarding legal protection. You are not allowed to alter this document in any way, to copy it for public or commercial purposes, to exhibit the document in public, to perform, distribute or otherwise use the document in public.

By using this particular document, you accept the above-stated conditions of use.

\section{Kontakt / Contact:}

\section{peDOCS}

DIPF | Leibniz-Institut für Bildungsforschung und Bildungsinformation Informationszentrum (IZ) Bildung

E-Mail:pedocs@dipf.de

Internet: www.pedocs.de

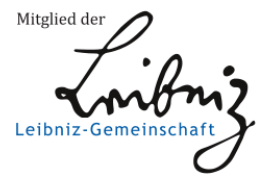


3. Jahrgang Heft 4

2014

บํํำ

$\stackrel{9}{9}$

$\frac{\Phi}{\partial}$

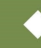

5

ฮ

เ্য

$\frac{1}{d}$

$\frac{\Phi}{0}$

ส্

อ

\)

4

ᄃ

$\Phi$

过

$\frac{\Phi}{c}$

हू

$\stackrel{\Delta}{2}$

$\pm$

E

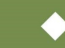

产

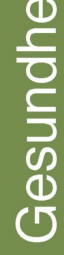

\section{Haushalt in}
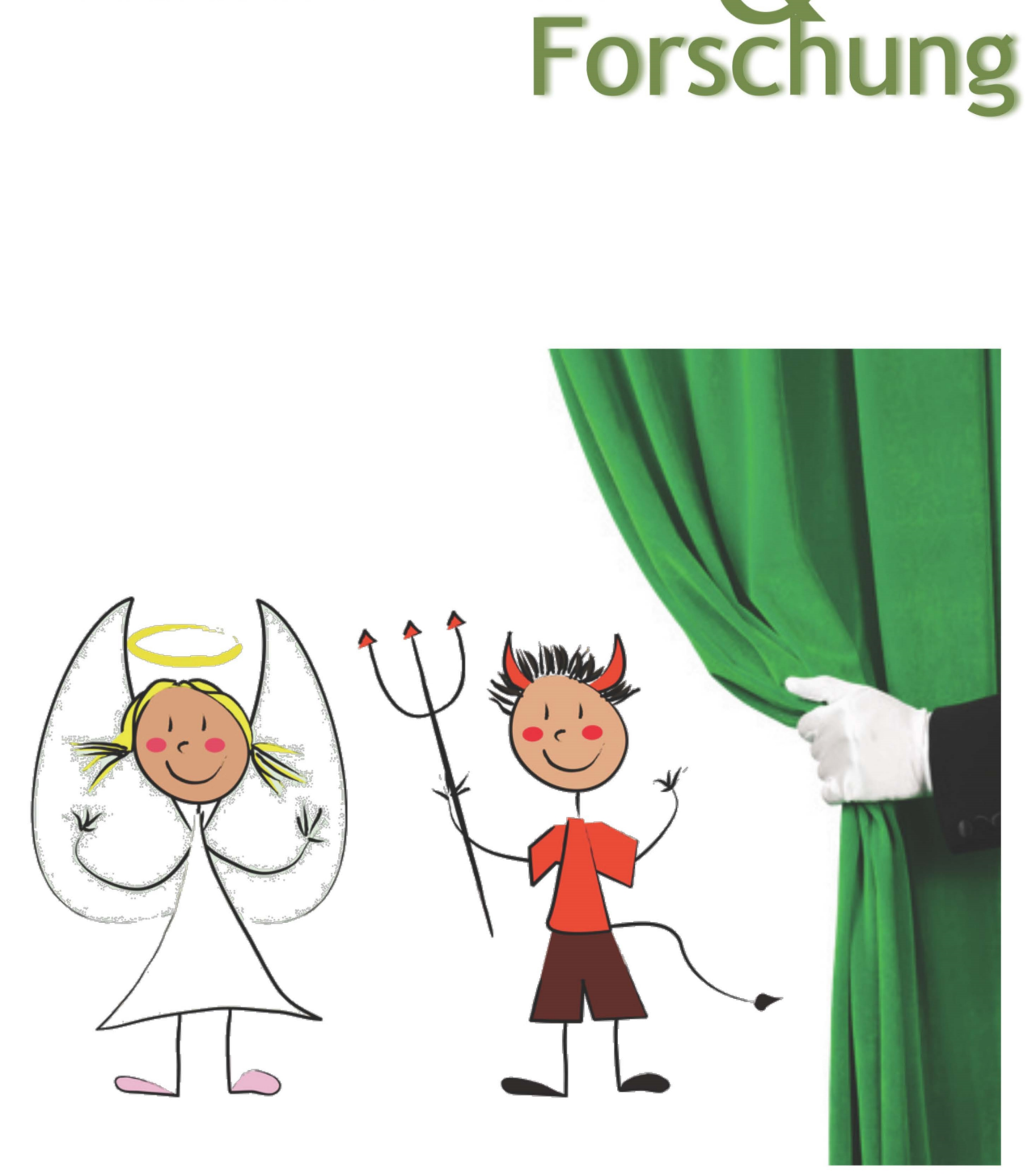

Du sollst! Du darfst! Ich muss? Zur Moralisierung von Ernährung und Gesundheit 
Inhaltsverzeichnis |

\section{Ursula Buchner}

Editorial

Ute Bender \& Anke Hertrampf

Fachbezogene moralische Überzeugungen von Lehrpersonen in der

Ernährungs- und Verbraucherbildung (EVB)

Claudia Suter \& Dominique Högger

Ernährungsbildung - was sollen, dürfen und müssen Lehrerinnen und Lehrer?.... 16

Heidrun Forstmaier

„Fit und gesund durch den Tag“ - Wie kann das bei der Auswahl

und Zubereitung von Lebensmitteln aussehen?.

Sonja Mannhardt

Du sollst, du musst, du darfst nicht: Agonie des Essens und des „guten

Geschmacks“......

Thomas Mohrs

Essen - Identität - Verantwortung. Konsumethische Reflexionen

Georg Raacke

„Wer früher stirbt, ist selbst schuld!“ - Ein kritischer Blick auf das aktuelle

Gesundheitsregime.

Gabriela Leitner

Erfolg M/m/acht Gesundheit

Werner Brandl

Und die Moral von der Geschicht'? - Über die Moralisierung der Moral............... 94

Einladung zur 3. D-A-CH-Fachtagung 2015 „Wa(h)re Gesundheit“...................... 104 
Was sollen, dürfen und müssen Lehrpersonen?

\section{Claudia Suter \& Dominique Högger}

\section{Ernährungsbildung - was sollen, dürfen und müssen Lehrerinnen und Lehrer?}

Die Kernforderungen nach gesunder Ernährung werden zu unreflektiert auf die Schule übertragen - auch von den Lehrerinnen und Lehrern selber. Ausgehend von einem allgemeinen Bildungsbegriff machen wir Vorschläge, wie eine Ernährungsbildung aussehen könnte, die sich mit angemessenen Erwartungen an die Schule und dem Sinnhorizont von Lehrpersonen verbinden lässt.

Schlüsselwörter: Public Health, Bildungsbegriff, Antinomie, überfachliche Kompetenz

\section{Die Erwartungen von Public Health}

Fragen rund um die Ernährung haben eine hohe Bedeutung für Public Health. Die Stiftung Gesundheitsförderung Schweiz investiert zusammen mit kantonalen Partnern einen Großteil ihrer Mittel in den Bereich ,gesundes Körpergewicht“ bei Kindern und Jugendlichen. Langfristiges Ziel der Stiftung ist es, den Anteil der Bevölkerung mit einem gesunden Körpergewicht zu erhöhen (Stamm et al., 2014; 4) bzw. bei Kindern und Jugendlichen ein nachhaltiges Bewusstsein für die Bedeutung einer ausgewogenen Ernährung, ausreichender Bewegung sowie eines gesunden Körpergewichts zu schaffen (Dubowicz et al., 2013; 8, 10).

Es finden sich keine Aussagen dazu, welcher Beitrag von den Schulen erwartet wird - auch nicht in den beiden von Gesundheitsförderung Schweiz veranlassten Monitoringberichten zur Arbeit der Schule (Meisen-Nussbaum et al., 2012, Dubowicz et al., 2013). Meisen-Nussbaum et al. (2012) evaluierten die „Vermittlung von Ernährungs- und Bewegungskompetenzen im Schulalltag“, definierten aber nicht, welche „Kompetenzen“ damit gemeint sind und wie eine entsprechende „Vermittlung“ zu verstehen und zu gestalten ist. In den Lehrplänen und Angeboten sowie in den Lehrpersonen-Interviews wurde nach dem „Thema“ oder dem „Bereich“ Ernährung gesucht bzw. gefragt (ebd.; 17, 21, 63). Die Erwartungen, die die Akteure von Public Health an die Lehrpersonen stellen, sind also bloß allgemein formuliert und bleiben insgesamt diffus.

Es greift unserer Meinung nach zu kurz, Ernährungsbildung nur als Summierung von Aussagen zu verstehen, die zu ihr eine thematische Nähe haben. Dies zu begründen und Ernährungsbildung differenzierter zu konzipieren, ist eines der Ziele dieses Beitrags. 


\section{Was sollen, dürfen und müssen Lehrpersonen?}

\section{Welche Erwartungen spüren die Lehrpersonen?}

Meisen-Nussbaum et al. (2012) erkennen insgesamt eine „Verunsicherung“: Die 20 befragten Lehrpersonen der 1. und 4. Klasse aus verschiedenen Schweizer Kantonen „sehen sich nicht als Experten auf diesem Gebiet und stellen unterschiedliche ,Ernährungswahrheiten ' bei den Eltern sowie den anderen Lehrpersonen fest. Hier fehlt ihnen die Fundierung des Themas, um einen eigenen gefestigten Standpunkt vertreten zu können" (ebd.; 7). Entsprechend sehen sich die Lehrerinnen und Lehrer nicht als Fachpersonen für Gespräche mit den Eltern (ebd.; 8). Bei den Fragen zu den Rahmenbedingungen und Handlungsspielräumen verwiesen die Lehrpersonen „oftmals auf den Lehrplan ..., aber den genauen Inhalt kannten nur die wenigsten“ (ebd.; 27). Allerdings ist diese Verunsicherung nicht spürbar, wenn die Lehrpersonen von den bearbeiteten Inhalten, den gewählten Methoden und insbesondere vom Wirkungspotential berichten: Sie beschreiben die Kinder als „sehr offen und empfänglich“ für das Thema Ernährung; in den 1. Klassen könne man „durch das Tun die Bewusstheit stärken und Begeisterung wecken“; in den 4. Klassen sei „schon ein hohes Niveau erreicht" (ebd.; 31).

Die Studie geht nicht darauf ein, wie diese positiven Berichte auf der einen Seite und die generelle Verunsicherung auf der anderen zusammenpassen. Wir vermuten, dass sich die Lehrpersonen für die konkreten Unterrichtssituationen ein eigenes, pragmatisches und aus ihrer Sicht stimmiges Handlungskonzept konstruiert haben. Um handlungsfähig zu sein, blenden sie allenfalls widersprüchliche oder zu anforderungsreiche Ansprüche aus. In der Interviewsituation sehen sich die Lehrpersonen jedoch dazu veranlasst, über ihr Handlungskonzept zu reflektieren und es zu rechtfertigen. Woher, wenn der Lehrplan nur den wenigsten bekannt ist, speisen sie in diesem Moment ihre Vorstellung, was von ihnen erwartet wird? Die festgestellte Verunsicherung legt nahe, dass sie nicht auf ein systematisch entwickeltes und reflektiertes Konzept, sondern ad hoc auf Alltagsvorstellungen zurückgreifen. Dabei dürfte die Thematisierung der Übergewichtsproblematik in der Öffentlichkeit die Interpretation der Lehrpersonen nicht unwesentlich beeinflussen. Denn die Erwartungen von Public Health an ein gesundes Ernährungsverhalten sind mithin Teil eines kollektiven Gedankengutes. Ebenso weit verbreitet wie das Wissen über gesunde Ernährung dürften demnach das schlechte Gewissen, teilweise auch die Gleichgültigkeit sein, mit denen man sich über das Wissen hinwegsetzt.

Die befragten Lehrpersonen haben wohl ihr Ernährungswissen sowie die persönlich erlebten und in ihrem Umfeld beobachteten Spannungen zwischen Wissen und Verhalten als wesentlichen Bezugspunkt vor Augen, wenn sie im Interview über Ernährungsbildung reflektieren. Zudem kennen sie wahrscheinlich entsprechende Spannungen auch von ihren Schülerinnen und Schülern, bringen sie mit deren Selbstbestimmungsrecht in Verbindung und fragen sich, inwiefern sie die persönliche Lebensführung der zu unterrichtenden Kinder bzw. deren Eltern zu beeinflussen 


\section{Was sollen, dürfen und müssen Lehrpersonen?}

befugt sind. Insofern spiegeln sich die geschilderten diffusen Erwartungen von Public Health in der Verunsicherung der Lehrpersonen.

\section{Ernährungsgrundsätze und Selbstbestimmungsrecht}

Hintergrund dieser Diffusität bzw. Verunsicherung ist eine grundlegende Schwierigkeit, nämlich die, dass Erwartungen bezüglich gesunder Ernährung mit dem Selbstbestimmungsrecht in einem Spannungsverhältnis stehen können. Die Akteure von Public Health sind sich dieser Spannung mindestens auf einer grundsätzlichen Ebene durchaus bewusst und beschreiben sie als Dilemma zwischen Chancengleichheit und Freiheit (Mattig, 2014).

In der Schule ist diese Schwierigkeit nicht auf die Ernährungsbildung beschränkt, sondern zeigt sich als immanentes Merkmal organisierter Bildung: Diese bedeutet für die Schülerinnen und Schüler in vielerlei Hinsicht Fremdbestimmung und sieht gleichzeitig in der Selbstbestimmung eines der wesentlichen Ziele. Helsper (2010, 34f.) bezeichnet dies als „Antinomie von Fremd- und Selbstbestimmung“, Antinomie verstanden als die gleichzeitige Gültigkeit ,einander eigentlich ausschließender Positionen“. Eng damit verbunden ist die „Sachantinomie“, verstanden als Spannung zwischen den Gesichtspunkten, die durch wissenschaftliche Erkenntnisse oder Lehrpläne legitimiert sind, und jenen, die ihre Bedeutung aus der Alltagswelt und Biographie der Lernenden gewinnen (Helsper, 2004; 78).

Wie gelingt es, diese Blockade zu überwinden und die Lehrpersonen in der Ernährungsbildung zuversichtlich und handlungsfähig zu machen? Die von MeisenNussbaum et al. (2012) befragten Lehrpersonen wünschen sich „Weiterbildungen mit motivierenden, glaubwürdigen Angeboten, die viel Praxis mit erprobten Inhalten berücksichtigen und trotzdem auch theoretische Aspekte und Hintergrundwissen ausgewogen vermitteln“ sowie „ein Ernährungsthemenangebot, in dem Materialien und aktuelles Wissen kindgerecht aufbereitet sind, das dann eins zu eins umgesetzt werden kann“ (ebd; 7). Die Lehrpersonen gehen davon aus, dass sich Ernährungsbildung mehr oder weniger standardisieren und in Weiterbildungen und Materialien vermitteln lässt. Diese Vorstellungen sind aus mindestens zwei Gründen fragwürdig. Erstens schätzen Lehrpersonen die Entscheidungs- und Gestaltungsfreiheiten ihres Berufsalltags; dass sie sich auf vorgegebene Modelle verlassen wollen, ist zumindest zweifelhaft. Zweitens lassen sich die beschriebenen Antinomien grundsätzlich nicht aufheben, sondern nur reflexiv handhaben (Helsper, 2004; 67); keine Form von Weiterbildungen und Materialien könnte dies den Lehrpersonen abnehmen.

Wir verfolgen deshalb einen anderen Ansatz und versuchen, die Ernährungsbildung weniger aus der Logik von Public Health, sondern aus jener von Schule und Bildung zu erschließen. 


\section{Was sollen, dürfen und müssen Lehrpersonen?}

\section{Bildung im Verständnis von Lehrpersonen}

„Der Diskurs über Bildung [ist] immer auch ein Diskurs über die Rolle des Menschen in der je gegenwärtigen Gesellschaft ... und zugleich über Anforderungen, die unter diesen Bedingungen an ihn und an die Pädagogik gestellt werden müssen“" (Durdel, 2002; 79). Angesichts der pluralistischen und individualistischen Gesellschaft scheint uns zum einen ein konstruktivistisches Verständnis von Bildung hilfreich zu sein. Stark verkürzt bedeutet dies, ,einen angemessenen Umgang mit Pluralität [zu] erlernen, mehrere Wahrheiten aushalten [zu] können [sowie] die Veränderung von Selbst- und Weltentwürfen angesichts der Konfrontation mit Fremdem“ (ebd.; 194). Zum anderen gehen wir aus von drei sich ergänzenden „Traditionslinien innerhalb des Bildungsdiskurses" (ebd.; 81):

- Bildung als „Selbstbewusstsein von Sozialisation“ verstanden als „kritische Subjektivität in der Auseinandersetzung mit dem gesellschaftlichen Umfeld“ (ebd.; 81);

- Bildung als „Ausrüstung ... im Sinne des Besitzes von Handwerkszeug ..., das es ermöglicht, sich in den kulturellen Gegebenheiten der Gesellschaft zurechtzufinden. Dazu gehören Komponenten wie die Beherrschung von Kultur- und Lerntechniken sowie das Verfügen über Wissen“" (ebd.; 81);

- Bildung als „Entwicklung von Persönlichkeitsdimensionen“ (ebd.; 82).

Welche Bedeutung haben diese Definitionen von Bildung für Lehrpersonen und ihr praktisches Handeln? Durdel stellt fest, dass „der Bildungsbegriff durch Lehrkräfte inhaltlich unterschiedlich gefüllt wird“ und „der Bezug zur Geschichte bzw. Theorie des Bildungsbegriffes ... nur interpretativ und nicht explizit" sei. Die drei genannten Traditionslinien seien jedoch „als zentrale Explikationen in den Bildungsbegriffsbestimmungen der interviewten Lehrkräfte zu finden“ (ebd.; 207), wenn auch dieser Rahmen durch pragmatische Bezüge zum Schulalltag „weiter und durchlässiger“ werde (ebd.; 208). Gleichzeitig wird dem individuellen Verständnis von Bildung „eine handlungsleitende, Sinn stiftende Funktion zugesprochen“ (ebd.; 207) und „ein schulinterner Diskurs über die inhaltliche Auslegung des Bildungsauftrages wird als eine gute Basis für die Verständigung über Ziel und Sinn pädagogischen Handelns betrachtet und als subjektive Entlastung wahrgenommen“ (ebd.; 209).

Das konstruktivistische Bildungsverständnis wurde den interviewten Lehrpersonen „als ein möglicher aktueller Bildungsbegriff angeboten“ und von diesen „als geeignet eingeschätzt, aktueller schulischer Arbeit Sinn und Richtung zu verleihen“ (ebd.; 209). Wir sind deshalb zuversichtlich, dass diese Überlegungen zum Bildungsverständnis nicht nur geeignet sind, Folgerungen für die Ernährungsbildung zu diskutieren, sondern dass diese Folgerungen auch grundsätzlich anschlussfähig sind an die Bedeutungen, die Lehrpersonen der Bildung im Allgemeinen und ihrer konkreten alltäglichen Tätigkeit im Speziellen geben. 


\section{Was sollen, dürfen und müssen Lehrpersonen?}

Allerdings erkennen die von Durdel interviewten Lehrkräfte im konstruktivistischen Bildungsbegriff auch sehr hohe Ansprüche, zum Beispiel

... wenn die Offenheit von Jugendlichen nicht bestehe, sich mit der Vielheit von Welt konfrontieren zu lassen. Die Lehrkräfte neigen dann mitunter dazu, diesbezügliche pädagogische Bereiche als Erziehungsarbeit und/oder als sozialpädagogisches Handlungsfeld zu typisieren, und koppeln die Prozesse von der Bildungsidee ab. (ebd.; 210)

Der konstruktivistische Bildungsbegriff versucht, solche Abkoppelungen zu vermeiden, indem er ein ganzheitlicheres Verständnis von Bildungsprozessen anbietet, „, in denen sowohl Lernvoraussetzungen als auch Prozesse des Selbst- und Weltverstehens miteinander verbunden werden können“ (ebd.). In dieser Lesart wären die beschriebenen Schwierigkeiten nicht als Hindernis, sondern als zusätzliche Begründung für derartige Bildungsprozesse zu verstehen.

\section{Folgerungen für die Ernährungsbildung}

Wir betrachten Ernährungsbildung in diesem Kontext, um sie als Teil der Bildungsidee und damit auch als Teil des Sinnhorizontes von Lehrpersonen verstehen zu lernen und sie angesichts der beschriebenen immanenten Schwierigkeiten mit angemessenen Handlungsmodellen und realistischen Erwartungen zu verbinden. Dazu gehen wir aus vom ,angemessenen Umgang mit Pluralität“ sowie vom „Selbstbewusstsein von Sozialisation“. Gerade mit Blick auf Ernährung ist Pluralität eines der wesentlichen Merkmale der Sozialisation von Kindern und Jugendlichen: angefangen bei den Ernährungsgewohnheiten am elterlichen Esstisch über die selektive Sichtbarkeit von Nahrungsmitteln und deren Herkunft im durchschnittlichen Alltag bis hin zum realen Angebot im Supermarkt. Ein angemessener Umgang mit Pluralität bzw. Bildung als „Selbstbewusstsein von Sozialisation“ bedeutet in diesem Zusammenhang, sich den gegebenen Einflüssen nicht einfach passiv auszusetzen, sondern selbstbewusst mitzuentscheiden, welchen Einflüssen man sich erstens aussetzen will und welche Bedeutung man zweitens einem bestimmten Einfluss geben will. Für die Schule heißt das mindestens dreierlei:

- Vielfalt an Erfahrungen anbieten, um die als selbstverständlich empfundenen Einflüsse zu ergänzen und Pluralität sichtbar und explizit zugänglich zu machen;

- Achtsamkeit gegenüber diesen Einflüssen und unserem Umgang damit schulen;

- Entscheidungskompetenz im Umgang mit den vorhandenen Einflüssen fördern.

Diese drei Vorschläge werden wir im Folgenden näher erläutern und für die Ernährungsbildung konkretisieren. 


\section{Was sollen, dürfen und müssen Lehrpersonen?}

\section{Vielfältige Erfahrungen rund um das Essen}

In vielen Kindergärten, teilweise auch noch in der 1. und 2. Klasse ist die gemeinsame Pausenverpflegung ein fester Bestandteil des Tagesablaufs. Schon kleine Kinder wissen, wie die gemeinsame Essenszeit abläuft. Das vermittelt Struktur und Sicherheit, fördert den Zusammenhalt. Viele Kinder erleben die Mahlzeiten in ihrem familiären Umfeld nicht mehr als verlässliche Größe im Tagesablauf. Die Erfahrung, dass Essen - neben der Ernährung - der Beziehungsbildung, dem gemeinsamen Genießen und der Kommunikation dient, geht verloren. Mit der gemeinsamen Pausenverpflegung erweitern Kindergarten und Schule das Erfahrungsspektrum der Kinder im Bereich Ernährung.

Dieser Aspekt wird auch von Public Health betont. Weshalb stellen wir ihn hier an den Anfang? Entscheidend scheint uns, mit welcher Haltung und Zielsetzung eine gemeinsame Zwischenmahlzeit gepflegt wird. Während Public Health die Gesundheit und damit konkrete Erwartungen an die Ernährungsweise ins Zentrum stellt, geht es uns viel basaler um vielfältige Erfahrungen. Auch wenn es in der praktischen Umsetzung viele Gemeinsamkeiten gibt, sehen wir in diesem Perspektivenwechsel wesentliche Vorteile: Lehrerinnen und Lehrer überwinden gegebene Verhaltenserwartungen und erweitern ihre Optik. Sie können in der Rolle von Bildungsfachleuten argumentieren und müssen sich nicht in die Rolle als Fachperson für Ernährung gedrängt fühlen. So geraten sie weniger ins Spannungsfeld zwischen Verhaltenserwartungen und dem tatsächlichem Verhalten (auch ihrem eigenen) und auch weniger in Konflikt mit den elterlichen Autonomieansprüchen. Als Modell müssen sie sich nicht in erster Linie ,gesund“ (wie immer das zu definieren wäre) verpflegen, sondern den Kindern vorleben, dass Essen vielfältig, abwechslungsreich und lustvoll ist, auch Süßes ohne schlechtes Gewissen gegessen werden darf. Es geht darum, einen natürlichen Umgang mit Essen vorzuleben.

Der eigentliche Akt der Verpflegung ist dabei eingebunden in einen weiteren Kontext: Manche Kindergärten bereiten ein- oder mehrmals in der Woche gemeinsam eine Zwischenverpflegung zu. Auch das Teilen und Tauschen ist eine Variante, Neues zu entdecken. Oft genug geschieht es, dass Kinder dabei bestimmte Nahrungsmittel zum ersten Mal kosten. Gemeinsam handelnd kommen Kinder und Lehrpersonen ungezwungen ins Gespräch über Vorlieben und Sachverhalte; zum Zug kommen darüber hinaus soziale Kompetenzen (gemeinsam essen und kommunizieren, Empathie beim Teilen, Verzichten etc.), Begegnungen mit Natur und Landwirtschaft (Anpflanzen und sich in Geduld üben, bis etwas geerntet werden kann), Entwicklung der Feinmotorik und Fertigkeiten im Umgang mit Nahrungsmitteln (Gemüse schälen, schneiden, zubereiten) etc.

Mit einem Pausenkiosk lässt sich das Thema Ernährung auch mit Jugendlichen auf der Erfahrungsebene angehen. Um einen solchen kompetent führen zu können, benötigen die Schülerinnen und Schüler entsprechendes Wissen, beispielsweise dar- 


\section{Was sollen, dürfen und müssen Lehrpersonen?}

über, welches saisonale Lebensmittel sind. Solche Inhalte bekommen so eine Relevanz, die ohne konkreten Handlungskontext nur schwer herzustellen ist.

\section{Weitere Erfahrungen durch Sach- und Selbstbezüge}

Dieses Beispiel macht deutlich, wie Sachbezüge konkrete Erfahrungen erweitern. Beim Thema Ernährung drängt es sich auf, den Blick zu weiten und Berührungspunkte zu anderen Themenfeldern zu suchen. Es geht dabei auch um Fragen der Herkunft unserer Lebensmittel, um Nachhaltigkeit, Konsum, Wirtschaft und Kultur. Das Thema lässt sich einbetten in den Sachunterricht ebenso wie in Geografie, Bildnerisches Gestalten, Bewegung und Sport, Hauswirtschaft und Geschichte. Die Thematik auf die physiologische Bedeutung der Lebensmittel zu reduzieren, greift zu kurz. Wiederum ist es uns wichtig zu betonen, dass wir damit keine bestimmten Verhaltenserwartungen verbinden, sondern primär vielfältige Erfahrungen anstreben, zumal Ernährungswissen das Verzehrsverhalten nur schwach beeinflusst (Schmid et al., 2012; 118). Sowohl ein „Zuviel“ an Informationen als auch verkürzte Merksätze erweisen sich als problematisch: Während ein „Zuviel“ tendenziell die Alltagswelt der Lernenden ignoriert und damit der erwähnten Sachantinomie nicht gerecht wird, fördern allzu starre Merksätze bei einem vermeintlichen Fehltritt eher das schlechte Gewissen sowie die Stigmatisierung von übergewichtigen Menschen. Einen hilfreichen Ansatz verfolgt u.E. das Lehrmittel „Pfefferkorn“ für die 1. bis 3. Klasse (Nold et al., 2002).

Darüber hinaus gehören auch Selbstbezüge zum Erfahrungsspektrum mit Essen und Ernährung. „Wie schmecken mir gewisse Nahrungsmittel?“”, „Welche Gefühle erlebe ich beim Essen?“”, „Wie nehme ich Hunger und Sättigung wahr?“, „Kann Essen trösten?“" oder „Fällt mir verzichten einfach?“ sind einige der Fragen, die sich im Zusammenhang mit Essen auf der einen, Selbst- und Gefühlswahrnehmung auf der anderen Seite stellen. Unter Selbstwahrnehmung verstehen wir die Fähigkeit, sich durch innere und äußere Wahrnehmung ein differenziertes Bild seiner selbst zu machen, sich als eigenständig wahrzunehmen und eigene Stärken und Schwächen zu kennen und zu akzeptieren. Selbstwahrnehmung beginnt beim Kennenlernen des eigenen Körpers und führt weiter zum Wissen um eigene Vorlieben, Stärken, Schwächen und Eigenheiten. Kinder lernen dadurch, sich so zu akzeptieren, wie sie sind, und sich nicht über den Vergleich mit anderen zu definieren. Sie werden darin gestärkt, eigene Bedürfnisse zu erkennen und diesen gemäß zu handeln. Auf den Bereich der Ernährung übertragen bedeutet dies beispielsweise, dass Hunger- und Sättigungsgefühle wahrgenommen werden.

Gefühlsbewältigung bezeichnet das Erkennen der eigenen Gefühle sowie deren Einfluss auf das eigene Verhalten und darüber hinaus die Fähigkeit, auch mit negativen Gefühlen angemessen umzugehen. Dies beinhaltet die Akzeptanz, dass unangenehme Gefühle zum Leben gehören und unter Umständen schwierig auszuhalten 


\section{Was sollen, dürfen und müssen Lehrpersonen?}

sind. Bezogen auf Ernährungsbildung ist etwa daran zu denken, dass Essen zu den Kompensations- und Bewältigungsstrategien von jenen Menschen gehört, denen der Umgang mit Gefühlen schwerfällt (Rytz et al., 2010; 13).

Das Lehrmittel „Körper und Gefühle im Dialog“ (Rytz et al., 2010) stellt diese beiden Kompetenzen gezielt in den Kontext Ernährung und präsentiert eine Sammlung von Materialien für Kinder von 4 bis 8 Jahren. Ernährung ist eng verbunden mit unserem Körper sowie unserem Alltag und daher ein sehr geeignetes Thema, um Selbst- und Gefühlswahrnehmung zu fördern. So verstandene Ernährungsbildung auf der einen und die allgemeine Förderung von Selbst- und Gefühlswahrnehmung auf der anderen Seite bedingen und begünstigen sich gegenseitig.

\section{Achtsamkeit gegen Einflüsse und den Umgang damit}

Vielfältige Erfahrungen mit Selbst- und Gefühlswahrnehmung können ihre Wirksamkeit vor allem dann entfalten, wenn es in konkreten Momenten gelingt, achtsam zu sein auf die Einflüsse, denen wir ausgesetzt sind, und auf unsere Handlungsimpulse im Umgang damit. Achtsamkeit „... beinhaltet, auf eine bestimmte Weise aufmerksam zu sein: bewusst, im gegenwärtigen Augenblick und ohne zu urteilen“ (Kabat-Zinn, 1998; 18). Eine achtsame Haltung ermöglicht uns, unser körperliches Befinden, unsere Gedanken und Gefühle im Moment des Auftretens zu erkennen. Dies führt zu Klarheit gegenüber unseren Bedürfnissen, Wünschen und Motiven: Das Wahrnehmen dessen, was im Moment geschieht, eröffnet einen Raum zwischen Reiz und Reaktion. Automatismen werden relativiert durch mehr Bewusstheit im Handeln. Dies kann dazu führen, dass Entscheidungen weniger impulsiv gefällt werden.

Achtsamkeit kann als Basiskompetenz betrachtet werden, auf der Lebenskompetenzen wie Selbstwahrnehmung, Gefühlsbewältigung und Entscheidungsfähigkeit aufbauen. Gleichzeitig ist die Achtsamkeitspraxis eine Methode, um diese angestrebten Fähigkeiten zu erlernen. Das Trainingsprogramm Mindfulness Based Stress Reduction (MBSR) von Jon Kabat-Zinn $(1998,2003)$ ist mittlerweile gut erforscht. Zunehmend interessiert sich auch die Pädagogik dafür, und es gibt verschiedene Programme für Kinder und Jugendliche. Im Zusammenhang mit Ernährung lässt eine achtsame Haltung Effekte auf mehreren Ebenen erwarten:

- Hunger- und Sättigungsgefühle werden durch die verbesserte körperliche Selbstwahrnehmung deutlicher. Körpersignale werden frühzeitig erkannt (z. B. Völlegefühl, Energiezunahme bzw. -verlust, Müdigkeit nach dem Essen).

- Der Umgang mit Essen ist weniger automatisiert (Kompensation, Tröster, Ersatz). Wir sind Gewohnheiten und Impulsen dadurch in geringerem Masse ausgeliefert und können uns leichter für dieses oder jenes Lebensmittel entscheiden. Der Raum zwischen Reiz und Reaktion eröffnet 


\section{Was sollen, dürfen und müssen Lehrpersonen?}

die Möglichkeit, sich bewusst für oder gegen etwas zu entscheiden (Belohnungsaufschub).

- Dank der Fähigkeit zur Selbstregulation lernen Kinder und Jugendliche, schwierige Situationen zu bewältigen, ohne auszuweichen oder zu verdrängen.

- Selbstwahrnehmung und bewusste Entscheidungen verringern die Gefahr, von Geboten, Verboten und Werbeversprechen abhängig zu sein, und vergrößern Freude und Genuss am Essen; Schuldgefühle und schlechtes Gewissen werden abgebaut bzw. vermieden.

\section{Reflexion und Entscheidungskompetenz}

Um Erfahrungen mit Achtsamkeitsübungen zu vertiefen und über die Übung hinaus wirksam werden zu lassen, bieten sich die Reflexion und das philosophische Gespräch an (zum Beispiel: Was heißt Genuss? Warum sind Vorlieben so unterschiedlich? Was wissen wir über die Herkunft von Lebensmitteln?). Während die Achtsamkeit einer Wahrnehmung im Hier und Jetzt eine Bedeutung zuweist und Raum schafft für die Reflexion, bezieht die Reflexion andernorts gewonnene (oder noch zu gewinnende) Erfahrungen mit Essen sowie deren Sach- und Selbstbezüge ein. Sie setzt verschiedene Aspekte in Beziehung und ermöglicht, allgemeine oder situationsspezifische Erkenntnisse als Entscheidungsgrundlagen abzuleiten.

Entscheidungsfähigkeit kann als zentrale Kompetenz im Umgang mit Vielfalt und Überfluss des Ernährungsangebotes betrachtet werden. Sie beinhaltet, die Notwendigkeit anstehender Entscheidungen zu erkennen, die Unsicherheit und Anspannung in unbestimmten Situationen auszuhalten, die relevanten persönlichen wie sachlogischen Gesichtspunkte zu erarbeiten, Handlungsvarianten zu entwickeln und mögliche Folgen in Erwägung zu ziehen sowie die getroffene Entscheidung zu begründen, auszuführen und im Rückblick zu reflektieren (Högger et al., 2012; 29). Dies macht deutlich, dass Entscheidungsfähigkeit auf Kompetenzen wie Achtsamkeit, Selbst- und Gefühlswahrnehmung und kritischem und kreativem Denken aufbaut.

Ernährungsfragen sind sehr geeignet, um Entscheidungsfähigkeit zu üben, aber nur ein Anlass unter vielen. Entscheidungsfähigkeit entwickelt sich über die ganze Schulzeit hinweg. Das beginnt bereits im Kindergarten damit, dass Kinder altersgemäße und für ihren Alltag relevante Handlungsoptionen erhalten sowie bewusst zu gestalten lernen, dass sie in sie betreffende Entscheidungen einbezogen werden und dass Lehrpersonen ihre eigenen Entscheidungen transparent machen und begründen. Die damit verbundenen Schwierigkeiten sehen wir dabei als zusätzliche Begründung dafür, erstens längerfristig und kontinuierlich am Aufbau von Entscheidungskompetenz zu arbeiten und zweitens spezifische Entscheidungskompetenz in Bezug auf einzelne Ernährungsfragen nicht isoliert, sondern im Kontext allgemeiner Bildung 


\section{Was sollen, dürfen und müssen Lehrpersonen? |}

und allgemeiner Entscheidungsfähigkeit zu verstehen. In letzter Konsequenz ist jede Förderung der Entscheidungskompetenz sowie der sie begründenden Basiskompetenzen wie Achtsamkeit, Selbst- und Gefühlswahrnehmung ein Beitrag zur Ernährungsbildung.

Damit berühren wir auch die beiden anderen erwähnten Traditionslinien des Bildungsdiskurses: Entscheidungskompetenz und ihre Grundlagen sind auch Teil einer Bildung als „Ausrüstung ... im Sinne des Besitzes von Handwerkszeug ..., das es ermöglicht, sich in den kulturellen Gegebenheiten der Gesellschaft zurechtzufinden“ (Durdel 2002; 81). Wird dies nicht nur als Technik verstanden, sondern ergibt sich daraus eine gewisse Gewöhnung und Haltung im Umgang mit den ,kulturellen Gegebenheiten der Gesellschaft“, ist dadurch auch die Bildung als „Entwicklung von Persönlichkeitsdimensionen“ (ebd.) berührt. In dieser Form lässt sich Ernährungsbildung mehrfach mit der Bildungsidee und damit auch mit dem Sinnhorizont von Lehrpersonen verknüpfen.

\section{Zusammenfassung und Fazit}

Was sollen, dürfen und müssen Lehrpersonen? Aus dem Gesagten fassen wir die folgenden Kernaussagen zusammen:

- Lehrpersonen sollen und dürfen den Mut haben, den Ansprüchen von Public Health an die Ernährungsbildung kritisch zu begegnen und sie im Kontext von Bildung und Schule eigenständig zu interpretieren.

- Um dazu einen gefestigten Standpunkt vertreten zu können, müssen sie nicht Fachpersonen für Ernährung sein, sondern dürfen sich auf ihre Expertise als Pädagoginnen und Pädagogen verlassen.

- Lehrpersonen dürfen nicht darauf hoffen, dass es Modelle der Ernährungsbildung gibt, die standardisierbar und frei von Antinomien sind und sich mit Hilfe von Lehrmitteln und Weiterbildung einfach kopieren lassen.

- Sie dürfen ihren eigenen Umgang mit Essen - gerade wenn er im Sinne von ernährungsphysiologischen Erwartungen nicht perfekt ist - als Ressource nutzen, um den Kindern eine lebendige Auseinandersetzung mit einem konkreten Stück Alltag zu ermöglichen.

- Sie sollen und dürfen Ernährungsthemen nutzen, um Selbst- und Gefühlswahrnehmung, Achtsamkeit und Entscheidungsfähigkeit zu fördern.

- Es darf von ihnen erwartet werden, dass sie überfachliche Kompetenzen als relevant erkennen und sie systematisch aufzubauen verstehen.

- Lehrpersonen dürfen sich von den Schwierigkeiten, die mit solchen Bildungsprozessen verbunden sind, nicht entmutigen lassen, sondern müssen versuchen, die Lernvoraussetzungen der Schülerinnen und Schüler mit den Prozessen des Selbst- und Weltverstehens zu verbinden. 


\section{Was sollen, dürfen und müssen Lehrpersonen?}

- Es gehört zur Professionalität von Lehrpersonen, mit Antinomien angemessen umzugehen.

- Lehrpersonen dürfen davon ausgehen, dass der (schulinterne) Diskurs über Bildung im Allgemeinen und solche Anforderungen im Speziellen die eigene Sinn- und Berufsorientierung immer wieder belebt.

Ernährungsbildung wird durch diese Vorschläge nicht einfacher. Aber sie lässt sich so mit angemessenen Handlungsmodellen und realistischen Erwartungshaltungen sowie dem Sinnhorizont von Lehrpersonen verbinden.

Damit ergeben sich auch Folgerungen für die Protagonisten von Public Health: Diese sollen sich nicht nur für das „Thema Ernährung“ in der Schule interessieren und stark machen, sondern für Bildung in einem umfassenden Sinne bzw. für jene Ausschnitte aus dieser erweiterten Perspektive, die zwar nicht auf den ersten Blick als Ernährungsbildung zu erkennen sind, aber deren Absichten längerfristig stützen und deren Erfolg mit bedingen. Dabei ist in Kauf zu nehmen, dass die Erwartungen von Public Health nicht im Sinne des Buchstabens erfüllt werden können. Wenn diese Abstriche nicht als Scheitern, sondern als das im Rahmen von Bildung Mögliche und Sinnvolle verstanden werden, lassen sich die diffusen Erwartungen an die Schule sowie die entsprechende Verunsicherung von Lehrpersonen eher überwinden.

Pädagogik war schon immer ein Ringen zwischen individuellen Freiheiten und standardisierten Erwartungen. Allerdings ist diese Kultur gerade angesichts von unzähligen gesellschaftlichen Forderungen an die Schule nicht einfach aufrechtzuerhalten. Ernährungsbildung ist zum einen darauf angewiesen, zum anderen ein möglicher Impuls dafür, dass das Ringen um angemessene Begründungen, Zielsetzungen, Herangehensweisen und Grenzziehungen lebendig bleibt.

\section{Literatur}

BAG: Bundesamt für Gesundheit (2013). Nationales Programm Ernährung und Bewegung. Bericht für die Jahre 2008-2012. Bern: BAG.

Dubowicz, A., Camerini, A., Ludolph, R., Amann, J. \& Schulz, P.J. (2013). Bewegung und Ernährung an Schweizer Schulen. Ergebnisse der zweiten Befragung von Schulleitungspersonen in der Schweiz und im Fürstentum Liechtenstein. Arbeitspapier 10. Bern und Lausanne: Gesundheitsförderung Schweiz.

Durdel, A. (2002). Der Bildungsbegriff als Konstruktion. Orientierungs- und handlungsleitendes Potenzial des Bildungsbegriffs. Hamburg: Kovač.

Helsper, W. (2004). Antinomien, Widersprüche, Paradoxien: Lehrerarbeit - ein unmögliches Geschäft? In B. Koch-Priewe, F. Kolbe \& J. Wildt (Hrsg.). Grundlagenforschung und mikrodidaktische Reformansätze zur Lehrerbildung (S. 4998). Bad Heilbrunn: Klinkhardt

Helsper, W. (2010). „Ich will, dass ihr selbständig werdet!“ Über die Widersprüche im Lehrerberuf. Friedrich Jahresheft, 28, 34-37. 


\section{Was sollen, dürfen und müssen Lehrpersonen?}

Högger, D., Suter, C., Rütti, A., Eglin, S. \& Suter S. (2012): Lebenskompetenz entwickeln. Eine Arbeitshilfe für die Schule. Aarau: Programm ,gsund und zwäg i de Schuel".

Kabat-Zinn, J. (1998). Im Alltag Ruhe finden. Freiburg: Herder.

Kabat-Zinn, J. (2003). Gesund durch Meditation. München: Barth.

Mattig, T. (2014). Autonomie als Herausforderung für die Gesundheitsförderung. Arbeitspapier 19. Bern und Lausanne: Gesundheitsförderung Schweiz.

Meisen-Nussbaum, G., Tettenborn Schärer, A. \& Zopfi, S. (2012). Evaluation der Vermittlung von Ernährungs- und Bewegungskompetenzen im Schulalltag. Forschungsbericht. Luzern: Pädagogische Hochschule Zentralschweiz.

Nold, D., Stuker, A., Gerber, G., Imhof, Ch. (2002). Pfefferkorn. Produzieren - Konsumieren. Hinweise für Lehrerinnen und Lehrer. Bern: Schulverlag.

Rytz, T., Uetz, R. \& Grandjean, V. (2010). Papperla Pep - Körper und Gefühle im Dialog. Bern: Schulverlag.

Schmid, A., Brombach, C., Jacob, S., Schmid, I., Sieber, R. \& Siegrist, M. (2012). Ernährungssituation in der Schweiz. Kapitel 2 des sechsten schweizerischen Ernährungsberichtes. Bern: Bundesamt für Gesundheit.

Schopper, D. (2010). „,Gesundes Körpergewicht“ bei Kindern und Jugendlichen. Was haben wir seit 2005 dazugelernt? Bern und Lausanne: Gesundheitsförderung Schweiz.

Stamm, H., Lamprecht, M. \& Wiegand, D. (2014). Monitoring zum Thema Gesundes Körpergewicht - Aktualisierung 2013. Arbeitspapier 20. Bern und Lausanne: Gesundheitsförderung Schweiz.

\section{Verfasserin/Verfasser}

Claudia Suter \& Dominique Högger

Beratungsstelle Gesundheitsbildung und Prävention

Pädagogische Hochschule FHNW

Bahnhofstr. 6

CH-5210 Windisch

E-Mail: claudia.suter@fhnw.ch, dominique.hoegger@fhnw.ch

Internet: www.fhnw.ch/ph/iwb/beratung/gesundheit 